\title{
Three-dimensional printing of atrial septal defect from echocardiographic images: feasibility, fidelity and potential applications
}

\author{
Dan'e Mei $^{1}$, jinling chen ${ }^{1}$, Qing Zhou ${ }^{1}$, Sheng $\mathrm{Cao}^{1}$, Zhiyu Zhao ${ }^{1}$, and Chuangli Feng ${ }^{1}$ \\ ${ }^{1}$ Wuhan University Renmin Hospital
}

November 2, 2021

\begin{abstract}
Objectives - We sought to investigate the technical feasibility, fidelity and the potential applications of three-dimensional(3D) printed atrial septal defect (ASD) models based on three-dimensional transesophageal echocardiography (3D-TEE) images preliminarily. Methods-We retrospectively collected 40 ASD patients' 3D-TEE images. The 3D-TEE data were imported into post-processing software to create printable $3 \mathrm{D}$ digital models, the patient-specific models were then printed by the $3 \mathrm{D}$ printer. Fidelity of the 3D printed models were quantitatively evaluated by comparing the measurements from 3D ASD models and echocardiographic data. Results - Ultrasound-derived 3D ASD models were acquired in all the forty cases successfully. There was good consistency and no significant differences in ASD size parameters among 3D printed models, 2D-TEE images and 3D-TEE images(all $P>0.05$ ). Also, it showed small difference among 3D printed models, 2D-TEE images and 3D-TEE images in the absolute difference value of ASD size parameters. There's a highly significant correlation between the ASD parameters including the maximal diameter, the minimal diameter and circumference measured by 3D ASD printed models and the corresponding parameters of ASD occluders applied in actual surgical procedure. Simulation exercises in the 3D ASD printed models had an impressive effect based on the comprehensive assessment of the ASD parameters. Conclusions-It is feasible to use 3D-TEE images as the data source of ASD 3D printed models. Ultrasound-derived ASD 3D printed models show highly fidelity, which contributes to provide evidence for the clinical application of 3D printing technology in decision of ASD occlusion.
\end{abstract}

\section{Introduction}

Three-dimensional (3D) printing technology, which was defined as the process of creating solid, 3D objects from a collection of images in the form of a digital file, has been applied to the medical field and played an increasingly significant role in the diagnosis and treatment of cardiac diseases due to the continuous advancement of materials, medical imaging technology, and the vigorous development of individualized cardiac therapy $^{1}$. As a bridge between physical and imaging data, the $3 \mathrm{D}$ printed models can display the complex intracardiac pathological anatomy with a more comprehensive, intuitive way and provide preoperative exercise opportunities for individualized surgery as well. Scholars have reported the application of 3D printing technology in the diagnosis and treatment of cardiac disease for such procedures as valve replacement and formation, complex congenital heart disease ${ }^{2,3}$.

CT (computed tomography) is the mainstream data source of 3D printing technology, but it may involve intravenous contrast and ionizing radiation, the image quality is affected by heart rate ${ }^{4,5}$. Three-dimensional echocardiography is another imaging modality with strong linear correlation with CT,new echocardiographic transducers and advanced software and hardware have optimized 3D echocardiographic images. Moreover, because of its portability, safety, none radiation exposure, high spatial and temporal resolution, superior 
ability to image fast moving structure ${ }^{6}$, involves no sedation or general anesthesia, echocardiography images may be more advantageous as the data source in the procedure of printing $3 \mathrm{D}$ models ${ }^{7,8}$.

Atrial septal defect (ASD) is one of the most common congenital heart diseases, accounting for $20 \%$ to $30 \%$ of adult congenital heart disease. As the effective therapeutic methods, ASD occlusion has been widely applied. Imaging data provided by different techniques always play a significant role in the diagnosis and treatment of ASD. However, they are lack of comprehensive, real touch and visual experience that can be obtained by a patient-specific physical model ${ }^{9}$. 3D printed models based on echocardiography images may show the anatomical details of the pathological structure clearly and act as the guidance for individualized treatment.

Feasibility and fidelity are prerequisites and guarantees for the ultrasound-derived 3D printing ASD models to be fully utilized. Whether it can highly retain and replicate the original image information is the basis for the 3D printed model to fully combine the advantages of three-dimensional transesophageal echocardiography (3D-TEE images) and 3D printing technology. Only when fidelity was guaranteed will the 3D printed model considered as an accurate measurement tool as the conventional 3D-TEE images and provide visual and tactile perception well. However, steps of creating 3D ASD model from an echocardiogram to a tangible 3D model are so complex that errors may be brought in, there is a risk of introducing design errors and manipulating the original source data in order to generate oversimplified $3 \mathrm{D}$ models ${ }^{10}$. Focused on processoriented outcomes, which means analysis the parameters in each step of the printing process, is an efficient method to assess the fidelity of the 3D models in some studies ${ }^{2,11}$.

This study aims to evaluate the feasibility and fidelity of 3D printed ASD models from echocardiographic images. Moreover, our study intends providing evidence for potential applications of 3D printing technology based on echocardiographic data in ASD therapy, especially individualized ASD occlusion.

\section{Materials and Methods}

\section{Patient population}

This was a retrospective study, patients who underwent closures of secundum ASD with the Amplatzer septal occluder (ASO, AGA Medical Corporation, Golden Valley, N, USA) successfully from March 2016 to February 2020 in Renmin Hospital of Wuhan University were enrolled in this study. The transesophageal echocardiography was performed by experienced investigators. Patients with arrhythmias, poor quality of transesophageal echocardiographic images or lack of complete clinical data as the size of ASO were excluded. A total of 40 patients had both 2D-TEE images and 3D-TEE images of high quality in our hospital database were finally considered for this study, of which 4 cases with double-hole, secundum ASD and 36 cases with isolated secundum ASD. All patients have complete clinical medical records. Baseline characteristics of the study population are reported in Table1. The study protocols were approved by the ethics committee at Renmin Hospital of Wuhan University. All patients or legal guardians gave their informed consent to study inclusion.

\section{TEE image acquisition}

Both 2D-TEE and 3D-TEE images were acquired by the GE Vivid E9 color Doppler platform (GE Vingmed Ultrasound AS, Horten, Norway) equipped with a 6VT transesophageal phased array probe during the procedure with the patients under anesthesia. Obtain two dimensional gray images of ASDs in 5 cardiac cycles when 2D-TEE clearly showed the cardiac defects in different planes from four-chamber $\left(0^{\circ}\right)$, short axis $\left(45^{\circ}\right)$, and bicaval $\left(110^{\circ}\right)$ view at mid-esophageal level. After which the $4 \mathrm{D}$ zoom function was applied to get the 3D-TEE images. Once the 4D mode was entered, 5 continuous root cycles volume images of the ASD were stored under single-beat mode. The desired 3D-TEE images should have completely dark blood pool and bright myocardial tissue with minimized noise in the blood pool and at the periphery of the data set. All the 2D-TEE and 3D-TEE images were exported to the EchoPAC workstation (GE Healthcare, WI) anonymously and the 3D-TEE images were converted to standard digital imaging and communications in medicine (DICOM) format (Fig. 1A ). 


\section{Image post-processing}

The DICOM format of high-quality 3D-TEE ASD images at the isovolumetric diastole stored in the EchoPAC workstation were imported into specialized post-processing software Mimics Innovation Suite 17.0 (Materialise, Leuven, Belgium), where the source imaging datasets can be converted to 3D digital models (Fig.1B ). Various post-processing methods including threshold segmentation, interactive segmentation and surface smoothing were used to create an accurate 3D digital ASD model. Firstly, threshold segmentation was applied to distinguish the blood pool border of the ASD from the endocardium, from which the blood pool could be filtered and the mask of the ASD were obtain initially. The threshold was adjusted with reference to the endocardial in different cases. After threshold segmentation, there still exist much noise and dropout gaps of the mask, manual editing is necessary to correct segmentations errors. Interactive segmentation was utilized to artificially exclude the noise and fill of the dropout gaps. Finally, surface smoothing function key was used to smooth the surface of the anatomy before exporting to the software 3-matic(Materialise, Leuven, Belgium), where the ASD mask could be calculated for a 3D hollow mold. (Fig. 1C )

\section{Creation of 3D printed ASD models}

The hollow model file was saved in standard tessellation language (STL). The printable STL files were imported into a high-precision 3D printer (JG AURORA Technology Co. Ltd, China, Shenzhen) to print the hollow 3D mold at a $1: 1$ scale with the soluble material polyvinyl alcihol (PVA). The rubber like material, which has been utilize in the previous studies of our laboratory ${ }^{12}$, was used for filling the 3D hollow ASD mold. Then, the hollow mold was dissolved with warm water and the individualized soft ASD 3D model was obtained. (Fig.1D)

\section{Analysis of ASD parameters}

The ASD parameters in this study including the maximum diameter, the minimum diameter, circular index (the ratio of the maximal to minimal diameters, ASD shape were distinguished by the circular index, round ASD defined as those patients with a circular index less than 1.4 and the oval shape defined as those with a circular index 1.4 or greater $)^{13}$, and circumference. All the measurements taken on the 2D-TEE images and 3D-TEE images were in the same portion of the cardiac cycle when the size appeared largest, typically isovolumetric diastole, which is the same frame image for post-processing and 3D printing. As to the measurement of 2D-TEE images, the bi-atrial view, the aortic root short axis view and the apical fourchamber view were regarded as the standard echocardiographic measurement section,the defect was measured in each of these views and the maximum diameter and the minimum diameter was determined. For 3DTEE measurements, the angle was carefully adjusted on the multiplanar reconstruction images to identify the largest plane, the longitudinal planes of the maximal and minimal ASD dimensions were identified using guidance on the en face image of the ASD, and the size parameters were measured on the longitudinal planes. When came to the 3D ASD models, the maximum diameter and the minimum diameter were measured by vernier caliper directly. A soft aluminum wire was used to shape the ASD, unfold the soft aluminum wire and the length measured by a vernier caliper was regarded as the circumference of ASD. All the measurements of ASD from the three methods were independently performed without knowing the results of others.

\section{Simulation operation in the $3 \mathrm{D}$ printed ASD models}

Five 3D printed ASD models were selected for the simulation exercises, of which four models were onehole ASD, one was double-hole ASD. The occlusion device utilized in this study was ASO, which is a self-expandable, double-disc, occlusion device made of nitinol mesh, approved by the US Food and Drug Administration in 2001 for percutaneous closure of secundum ASD ${ }^{14}$. Based on the soft ASD 3D models, the desired occluder was determined, the appropriate occluder applied in vitro trial occlusion was defined as the smallest one that occluded the ASD completely,kept stable and not oppressed in push-pull test. Obtain the patients' occluder size actually applied during the ASD occlusion by querying the clinical medical record system retrospectively. Evaluate the effect of simulation operations and assess whether the size of the optimized occluder required in the simulation exercise is consistent with the one actually applied during the ASD occlusion. 


\section{Observer variability}

Intra- and interobserver variability of the ASD measurements were assessed in 5 randomly selected subjects. For interobserver variability, the measurements were analyzed by two different observers independently in a randomized double-blind trial. For determining intraobserver reproducibility, the data were analyzed two days apart by the same observers who were blinded to the final results from their first analysis.

\section{Statistical analysis}

Statistical analyses were performed using SPSS version 17.0 (SPSS, Chicago, IL) and MedCalc version 11.0.1.0 (MedCalc Software, Mariakerke, Belgium). A P valuej0.05 was considered to indicate statistical significance. Continuous variables are expressed as mean+-standard deviation (SD) and categorical variables as numbers or percentages. All data were first analyzed to assess the normality of their distribution using Kolmogorov-Smirnov tests. Paired sample t tests were used for comparisons of measurements between 3D ASD models and 2D-TEE images, 3D ASD models and 3D-TEE images. A Bland-Altman analysis in MedCalc statistical software was performed for investigate consistency of the ASD size parameters measured between 3D models and 3D-TEE images, as well as measurements between 3D models and 2D-TEE images. Correlations between the size of ASD occluder applied intraoperative and the ASD parameters measured from 3D printed ASD models were determined by linear regression analysis. Interobserver and intraobserver agreement were analyzed by the intraclass correlation coefficient (ICC), a p values below 0.05 were considered statistical significance.

\section{Results}

\section{Feasibility of ultrasound-derived 3D printed ASD models}

3D-TEE images of all the 40 ASD patients were successfully post-processed, and then the corresponding 3D models of ASD were printed by the high-precision fused deposition modelling (FDM) printer. The total time from data set acquisition to standard tessellation language (STL) file generation and final 3D model printing was approximately 16 hours. All the 3D printed models were translucent, smooth and soft (Fig 2 ),36 models with one-hole secundum ASD and 4 models with double-hole secundum ASD, visual inspection showed good agreement between the 3D models and the TEE data sets.

\section{Comparison of the measurements among 2D-TEE images, 3D-TEE images and 3D printed models of ASD}

There was no significant difference in ASD size parameters among 2D-TEE images, 3D-TEE images and 3D printed models of ASD (all $P>0.05)$. (Table 2 and Table3 )

Bland-Altman scatter plot showed that the ASD size parameters were concordant well between 3D printed models and 2D-TEE images, the limits of agreement for the maximum diameter, the minimum diameter and circular index of ASD were -1.17 and $1.33 \mathrm{~mm},-1.19$ and $1.34 \mathrm{~mm},-0.16$ and 0.16 . Points within the range of consistency boundaries account for $92.5 \%, 100 \%$ and $92.5 \%$, respectively. (Fig. 5 ) Moreover, the agreement between the 3D printed models and 3D-TEE images measurements, which were assessed on the basis of Bland-Altman analysis, showed that the limits of agreement for the ASD parameters as the maximal diameter, minimal diameter, circular index and circumference were -1.16 and $0.95 \mathrm{~mm},-1.31$ and $1.21 \mathrm{~mm},-0.14$ and $0.13,-2.9$ and $2.4 \mathrm{~mm}$, respectively. The majority of plots $(95 \%, 95 \%, 96.25 \%$ and $100 \%$, respectively) fell within the limits of agreement. (Fig. 4 )

The absolute difference of the measurements among 2D-TEE images, 3D-TEE images and 3D printed models of ASD

The absolute differences of maximum diameter and the minimum diameter between 3D printed models and the 2D-TEE images were $0.51+-0.39 \mathrm{~mm}, 0.54+-0.35 \mathrm{~mm}$, respectively. The absolute differences of the maximum diameter, minimum diameter and circumference measured from 3D printed models and 3D-TEE images were $0.43+-0.33 \mathrm{~mm}, 0.52+-0.38 \mathrm{~mm}, 1.21+-0.66 \mathrm{~mm}$, respectively. Indicating that the 3D printed 
ASD models can retain and copy the original information of the source images, and no intolerable errors have been introduced during the complicated printing process.

Correlation analysis for the measurements of 3D printed models and occluder device size in ASD occlusion therapy

All of the 40 patients underwent ASD occlusion therapy in Renmin Hospital of Wuhan University successfully. 20 patients had round shape ASD and 20 patients had oval shape ASD. There was significant correlation between 3D printed models and the occluder device actually applied intraoperative in the maximum diameter, the minimum diameter and circumference data, whose Pearson correlation coefficient were $0.88,0.87,0.90$, respectively(all pi0.05) . Compared with the other three parameters, circumference of the 3D printed models showed a more significant correlation with the occluder device size $(\mathrm{p} i 0.05)$. The $3 \mathrm{D}$ printed ASD size parameters and the intraoperative occluder size parameters were shown in Table 4 .

\section{Simulation operation of ASD occlusion in 3D printed models}

Simulation operation of ASD occlusion was performed in five patients' 3D printed models. Three cases succeeded in simulation operation the first time, while two finished simulation operation the second time.

\section{(Table 5)}

In one case, the ASO was implanted successfully in the second try because of its large round shape. The maximal diameter, the minimum diameter and circumference measured from the 3D printed ASD model was $22.62 \mathrm{~mm}, 20.67 \mathrm{~mm}, 71.19 \mathrm{~mm}$, respectively. The circular index defined as the ratio of its maximal to minimal diameters was 1.1(i1.4). According to the principle of conventional clinical selection of the ASD device size, a $28 \mathrm{~mm}$ ASO was delivered into the ASD model, however the occluder is so small that result in residual leak. As a result, a $32 \mathrm{~mm}$ ASO was selected in the second attempt, the model-matched occluder was placed in 3D printed ASD model successfully with the waist of occluder slightly compressed and the double-discs paralleled with the ASD plane

\section{(Fig.3 A)}

In another case, which was the biforate ASD and had $6 \mathrm{~mm}$ apart between the two holes, the defects were closed by a single occluder device. The maximal diameter of the two defects and the whole size that measured from the 3D printed ASD model was $16.09 \mathrm{~mm}, 6.68 \mathrm{~mm}$ and $21.28 \mathrm{~mm}$, the minimum diameter of each hole and the whole size was $10.00 \mathrm{~mm}, 5.17 \mathrm{~mm}$ and $18.47 \mathrm{~mm}$, respectively. During simulation operation, occlusion was simulated in vitro using the 3D biforate ASD model to inform the closure plan, including the determination of target defect and size selection of the device. After the failure attempt with a $30 \mathrm{~mm}$ occluder, a $34 \mathrm{~mm}$ ASO was finally selected to cover all the defects successfully with the bigger defect as the optimal target defect. (Fig3 C )

In the other three cases, the size of the ASO devices selected in simulation operation according to 3D printed ASD models were identical with that applied in actual surgical procedure obtained by checking the medical records retrospectively. Moreover, simulation operation in 3D printed ASD model all showed no device replacement and occlusion-related complications such as residual leaks or device migration immediately after the in vitro simulation. (Fig.3 )

\section{Observer variability}

Intraobserver ICC for the ASD parameters as the maximal diameter, the minimal diameter and circular index via $2 \mathrm{D}$-TEE were $0.96,0.93,0.94$, respectively. Intraobserver ICC for the maximal diameter, the minimal diameter, circular index and circumference values based on 3D-TEE images were 0.98, 0.92, 0.98 and 0.93, respectively. The Intraobserver ICC for the corresponding parameters measured from 3D printed models were $0.97,0.92,0.99$ and 091 , respectively. Interobserver ICC for the same parameters based on 2D-TEE were $0.93,0.90,0.98$, respectively, and via 3D-TEE were $0.98,0.94,0.98,0.90$, respectively. Interobserver ICC of the maximal diameter, the minimal diameter, circular index and circumference based on 3D printed model were $0.95,0.90,0.98$ and 089 , respectively. 


\section{Discussion}

This study accessed the feasibility and the fidelity of 3D printed ASD models obtained from 3D-TEE data sets. The major findings of this study were that (1) Through image acquisition, post-processing, the corresponding ASD 3D models which have the same morphological structure with 3D-TEE data sets could be obtained effectively, it was technically feasible to use 3D-TEE images as the data source of 3D printing ASD models. (2) The parameter information of data source images could be highly replicated and retained by the 3D models under strict error control. Ultrasound-derived ASD 3D printed models had highly fidelity. (3) The 3D printed models derived from ultrasound may help plan the ASD closure surgery and make an informed selection of appropriate device.

\section{Advantages of 3D printed ASD models based on 3D-TEE images}

The clinical diagnosis and treatment of Atrial septal defect (ASD) mainly depends on echocardiography images. But the 2D images often lack of pivotal spatial information and fully measurable morphological parameters, higher requirements are raised for the cardiologists and cardiovascular surgeons to a mental conversion of $2 \mathrm{D}$ data into a $3 \mathrm{D}$ understanding of the spatial relationships of intracardiac structures ${ }^{9}$. Although 3D images can show the morphology of ASD more comprehensive, in patients with complex types such as ASD with atrial septal aneurysm (ASA), ASD with multi holes, ASD of inferior vena cava type and ASD with rim deficiency, 3D-TEE has been less beneficial for the intuitive and accurate surgical operation plan. The emerging 3D printing technology is a process that a solid, patient specific physical 3D model is created through layer-by-layer superposition molding from rendered computer-generated images, which makes it possible to create patient-specific 3D models based on imaging data ${ }^{15}$. These tangible models of patient anatomy have demonstrated value in education, simulation, and surgical planning ${ }^{16}$. 3D printed ASD models can not only provides a more intuitive 3D perspective, comprehensively shows the morphological structure of the lesion to improves the diagnostic accuracy, but also provides haptic feel, enabling the surgeon to a more realistically understanding for the deformation of the surgical area produced by external pressure during the operation and help plan the surgical approach to enhance the success rate of the treatment ${ }^{17,18}$. The size and shape of ASD can be observed intuitively, and the simulated interventional treatment can be performed on the model, which may provide more information for the therapy.

Compared with $\mathrm{CT}$, which is the most commonly used medical imaging modalities for 3D image generation, echocardiography has a superior ability to image fast moving structures such as cardiac valves and intracardiac defect and directly display the area of interest and intracardiac defects with high image resolution ${ }^{6}$. Moreover, 3D-TEE is an important imaging method that plays a critical role in the process of preoperative diagnosis, intraoperative monitoring and postoperative efficacy evaluation of ASD. Using 3D-TEE images as the data source of 3D printed ASD models can combine the advantages of echocardiography and 3D printing technology effectively.

\section{Feasibility and fidelity of 3D printed ASD models based on 3D-TEE images}

Feasibility and fidelity are significant prerequisites and guarantees for the medical application of ultrasoundderived 3D printed models. However, steps involved in creating a 3D printed model were meticulous and complex. Errors are easily introduced in the sophisticated workflow of image acquisition, image post-processing and 3D model manufacture, which may decrease the fidelity of 3D models. Therefore, the ability to highly retain the information of original 3D-TEE images is the key for the 3D models to combine the advantages of 3D-TEE and 3D printing technology. Quantitative assessment of fidelity is of great significance for subsequent application in ultrasound-derived $3 \mathrm{D}$ printing ${ }^{11}$.

In this preliminary study, all the 3D-TEE ASD images were successfully post-processed and converted to solid 3D ASD models, demonstrating the feasibility of echocardiography used as the data source for the 3D printing of models, which requires a trained echocardiographer, a 3D echocardiography system, a postprocessing software for data conversion, segmentation, modeling and a 3D printer ${ }^{19}$. The first condition for successfully obtaining 3D ASD models is to acquire the 3D-TEE images and converted to a DICOM format that can be accessed by offline post-processing software. The ultrasound systems used in this study are 
capable of exporting 3D data in the DICOM format, in which the echocardiographic images can dynamically display the real-time changes of ASD in each phase during the cardiac cycle. By replaying the dynamic characteristics of the observed images, the key frames at the isovolumetric diastole are selected for image post-processing. In addition, whether ultrasound-derived 3D printing is feasible depends on post-processing software. Mimics software applied in this study is one of the available post-processing software that have a wide selection of manual and automatic segmentation tools and capable of correcting mesh files, which allowing to obtain patient-specific modelling and meshes ${ }^{19}$. The last basis for 3D printing is 3D printer, the FDM 3D printer used in our study can generate instructions and build the 3D ASD model layer by layer completely.

ASD occlusion is considered to be the first-line therapy for patients with morphologically suitable secundum $\mathrm{ASD}^{20}$. For achieving successful closure of ASD, accurate determination of the device size is one of the critical steps in the procedure. The maximum diameter is considered to be the most important reference parameters in choosing the appropriate size of an ASD occluder with central waist ${ }^{21}$. However, the shape of the ASD is oval or irregular rather than round occasionally, self-expansion properties of the ASO may cause deformation of the ASD. Noticeable changes may generate in the maximum diameter and the minimum diameter of ASD simultaneously and the degree of change depends on the shape of ASD. Studies have pointed out that stretching of the oval ASD by the device will probably occur by preference at the minimum dimension and make the ASD more circular ${ }^{22}$. A single maximal diameter measurement might overestimate the device size in patients with an oval, large ASD and cause the selection of devices oversized ${ }^{23}$. Considering that the maximum diameter and the minimum diameter will change with the implantation of the occluder, the parameters that do not change with deformation should also be analyzed, especially the circumference. The circumference may be a meaningful reference to estimate device ${ }^{13}$.

Therefore, parameters measured from 3D printed models in this preliminary study have reference significance for ASD closure, such as the maximal diameter, the minimal diameter, circular index and circumference. It showed no significant differences among the three methods. What's more, the Bland-Altman scatter plot demonstrates that the ASD size parameters were concordant well among 3D printed models,2D-TEE images and 3D-TEE images. Moreover, the absolute difference was small among the three methods as well. All these results indicate that the 3D printed ASD models precisely preserve the original information of the ultrasound images and the fidelity of ultrasound-derived 3D ASD models can be guaranteed if meticulous care is taken in the workflow. 3D printed models have the potential to reflect the ASD anatomy accurately.

This preliminary study is focused on process-oriented outcomes to evaluate whether tiny errors were introduced in every step. The high fidelity of the ASD 3D printed models mainly depend on tight error control during data acquisition, image post-processing and printing described herein ${ }^{24}$. Firstly, the acquisition of optimized 3D-TEE ASD images is the basis for accurate 3D printed models. Newer echocardiography transducers, coupled with the continuous advanced ultrasound hardware and software equipment makes it possible to obtain 3D echocardiographic images with high resolution. The grayscale variations within the structure of interest were minimized and the contrast between ASD and surrounding area were maximized. The desired ASD 3D echocardiographic data set we finally obtained have a completely dark blood pool and completely bright myocardium with minimal noise and motion blurring. Secondly, the keys for a precision post-processing are to recognize fine details of ASD and adjust the thresholding to the optimal. During post-processed, the upper and lower limitation of thresholding was altered according to the endocardium meticulously. There were no cases of missing ASD structures or excessive image noise caused by improper threshold settings and manual segmentation errors. The balance between signal and noise of the 3D ASD digital models were maintained and the important anatomic details of ASD were saved well. Thirdly, the fidelity of the 3D printed models also benefited from the high resolution of the printer and its advanced control software. FDM printers can provide high resolution ranging from 0.1 to $0.2 \mathrm{~mm}$ after considerable post-processing, tiny errors in the process of $3 \mathrm{D}$ model manufacture can be avoided effectively ${ }^{24}$. In this study, quantitative comparisons of the results with the original data set at every stage of processing were taken carefully. It can be confirmed that the $3 \mathrm{D}$ printed model can highly retain and reproduce the original 3D-TEE image information. 


\section{Potential applications of ultrasound-derived 3D printed ASD models in ASD occlusion}

AS an established alternative to surgical repair, closure surgery of secundum ASD still remains some challenges. The appropriate device can't always be predicted accurately in advance. Replacement of the occluder occurs occasionally during the operation if the estimated one is too large or too small. Furthermore, in patients with multiple defects, the identification of appropriate candidates for closure and the selection of surgical approach are important. Though single device in the largest defect to occlude the entire defect remains optimal strategy ${ }^{25}$, it is still technically challenging because of inability to determine the target defect for catheter passage and occluder selection pre-operation, which highlight the necessity of comprehensive evaluation of the ASD parameters and preoperative simulation operation for successful device closure. This study showed a high correlation between the ASD parameters measured from the 3D printed ASD models and the size parameters of intraoperative occluders, among which the circumference of 3D printed models resulted to be most relevant to the occluder device size. 3D printed ASD models can mimics the "en face" view for evaluating the morphological characteristics of an ASD comprehensively and provide reference for determine individualized plans in ASD occlusion ${ }^{26}$.

In this preliminary study, the rubber-like material was used to make the 3D models, it can mimics the deformation of ASD structure after device implantation well. On this basis, the ASD 3D printed models were utilized as an intuitive tool for selection of desired occluder during the experiments in vitro. The device size was adjusted based on the parameter measured from 3D printed models and changes of morphological of ASD after the ASO implantation. As a result, the size of ASO selected according to the simulation operation was consistent with the one that actually applied in individualized ASD occlusion. In this simulation operation, if only taking the maximal diameters into consideration, the devices would be undersized or oversized. Associated with the correlation analysis results, other parameters as the minimum diameter and circumference were also taken into account. As a result, the desired occluders were implanted in the 3D printed models successfully, which had the same size as the one utilized in actual operation. In biforate ASD case, the whole size and shape of the two holes were the reference to the determination of appropriate occlude, the optimal target defect and device size was selected through several experiments in vitro in the tangible-patient specific model. The simulation operation indicated that the 3D printed ASD model based on echocardiography has a great potential for integration into clinical practice to assist with device selection and decision-making.

\section{Limitations}

The 3D printed models of ASD are static that can't stimulate the dynamic characteristics of the intracardiac anatomy intuitively and conveniently. In this initial research, the surrounding structures of ASD that have significant importance were not analyzed exhaustively, the parameters reflecting the surrounding structures should be included in the future research.

\section{Conclusions}

It is technically feasible to print 3D models of ASD from echocardiographic data. 3D printed model of ASD based on echocardiographic has high precision and can accurately retain the characteristics of original data source images. This study may lay foundation for the application of 3D printed models in procedural planning and size selection for ASD occlusion.

Acknowledgment This work was supported by Teaching and Research Project of Wuhan University School of Medicine (No. 2021014). The authors wish to thank everyone who helped us during this research.

\section{Compliance with ethical standards}

Conflict of interest All authors have no conflict of interest to declare.

Ethical approval All procedures performed in studies involving human participants were in accordance with the ethical standards of the Institutional Review Board of the Einstein Healthcare Network and with the 1964 Helsinki declaration and its later amendments or comparable ethical standards. 
Informed consent Informed consent was obtained from all individual participants included in the study.

\section{Reference}

1. Sarris GE, Polimenakos AC.Three-Dimensional Modeling in Congenital and Structural Heart Perioperative Care and Education: A Path in Evolution. Pediatr Cardiol 2017;38:883-885.

2. Mowers KL, Fullerton JB, Hicks D, et al. 3D Echocardiography Provides Highly Accurate 3D Printed Models in Congenital Heart Disease. Pediatr Cardiol 2021;42:131-141.

3. Loke YH, Harahsheh AS, Krieger A, et al. Usage of 3D models of tetralogy of Fallot for medical education: impact on learning congenital heart disease. BMC Med Educ 2017; 17:54.

4. Ghisiawan N, Herbert CE, Zussman M, et al. The use of a three-dimensional print model of an aortic arch to plan a complex percutaneous intervention in a patient with coarctation of the aorta. Cardiol Young 2016;26: 1568-1572.

5. Bhatla P, Tretter JT, Ludomirsky A, et al. Utility and Scope of Rapid Prototyping in Patients with Complex Muscular Ventricular Septal Defects or Double-Outlet Right Ventricle: Does it Alter Management Decisions? Pediatr Cardiol 2017; 38: 103-114.

6. Byrne N, Velasco FM, Tandon A, et al. A systematic review of image segmentation methodology, used in the additive manufacture of patient-specific 3D printed models of the cardiovascular system. JRSM Cardiovasc Dis 2016; 5:401155419.

7. Faganello G, Campana C, Belgrano M, et al. Three dimensional printing of an atrial septal defect: Is it multimodality imaging? Int J Cardiovasc Imaging 2016;

32:427-428.

8. Vukicevic M, Faza NN, Avenatti E, et al. Patient-Specific 3-Dimensional Printed Modeling of the Tricuspid Valve for MitraClip Procedure Planning. Circulation: Cardiovascular Imaging 2020;13: e010376.

9. Luo H, Xu Y, Wang Z, Liu Y, et al. Three-Dimensional Printing Model-Guided Percutaneous Closure of Atrial Septal Defect. Arq Bras Cardiol 2017;108:484-485.

10. Mathur M, Patil P, Bove A. The Role of 3D Printing in Structural Heart Disease: All That Glitters Is Not Gold. JACC Cardiovasc Imaging 2015; 8: 987-988.

11. Muraru D, Veronesi F, Maddalozzo A, et al. 3D printing of normal and pathologic tricuspid valves from transthoracic 3D echocardiography data sets. Eur Heart J Cardiovasc Imaging 2017;18:802-808.

12. Dan Jia, Qing Zhou, Hong-ning Song, et al. The value of the left atrial appendage orifice perimeter of 3D model based on 3D TEE data in the choice of device size of LAmbre occlude. The International Journal of Cardiovascular Imaging, 2019;35:1841-1851.

13. Hascoet S, Hadeed K, Marchal P, et al.The relation between atrial septal defect shape, diameter, and area using three-dimensional transoesophageal echocardiography and balloon sizing during percutaneous closure in children. Eur Heart J Cardiovasc Imaging 2015;16:747-755.

14. Turner DR, Owada CY, Sang CJ, et al. Closure of Secundum Atrial Septal Defects With the AMPLATZER Septal Occluder: A Prospective, Multicenter, Post-Approval Study. Circ Cardiovasc Interv 2017;10:e004212.

15. Smerling J, Marboe CC, Lefkowitch JH, et al. Utility of 3D Printed Cardiac Models for Medical Student Education in Congenital Heart Disease: Across a Spectrum of Disease Severity. Pediatr Cardiol $2019 ; 40: 1258-1265$. 
16. Zhu Y, Liu J, Wang L, Guan X, et al. Preliminary study of the application of transthoracic echocardiography-guided three-dimensional printing for the assessment of structural heart disease. Echocardiography 2017;34:1903-1908.

17. Ma Y, Ding P, Li L, et al.Three-dimensional printing for heart diseases: clinical application review. Biodes Manuf 2021:1-13.

18. He L, Cheng GS, Du YJ, et al. Feasibility of Device Closure for Multiple Atrial Septal Defects With an Inferior Sinus Venosus Defect: Procedural Planning Using Three-Dimensional Printed Models. Heart Lung Circ 2020;29:914-920.

19. Mashari A, Montealegre-Gallegos M, Knio Z, et al. Making three-dimensional echocardiography more tangible: a workflow for three-dimensional printing with echocardiographic data. Echo Res Pract 2016;3:R5764.

20. Nakayama R, Takaya Y, Akagi T, et al. Efficacy and safety of atrial septal defect closure using Occlutech Figulla Flex II compared with Amplatzer Septal Occluder. Heart Vessels 2021;36:704-709.

21. Kitakata H, Itabashi Y, Kanazawa H, et al. Appropriate device selection for transcatheter atrial septal defect closure using three-dimensional transesophageal echocardiography. Int J Cardiovasc Imaging 2021;37:1159-1168.

22. Boon I, Vertongen K, Paelinck BP, et al. How to Size ASDs for Percutaneous Closure. Pediatr Cardiol 2018;39: 168-175.

23. Jang JY, Heo R, Cho MS, et al.Efficacy of 3D transoesophageal echocardiography for transcatheter device closure of atrial septal defect without balloon sizing. European Heart Journal - Cardiovascular Imaging 2018;19:684-689.

24. Meier LM, Meineri M, Qua HJ, et al. Structural and congenital heart disease interventions: the role of three-dimensional printing.Neth Heart J 2017;25:65-75.

25. Sa YK, Park CS, Cho EJ, et al. Single device transcatheter closure for double atrial septal defect under real time three-dimensional image guidance [published online ahead of print Jul 15 ,2021]. The Korean Journal of Internal Medicine. doi: 10.3904/kjim.2021.094.

26. Yan C, Wang C, Pan X, et al.Three-dimensional printing assisted transcatheter closure of atrial septal defect with deficient posterior-inferior rim. Catheter Cardiovasc Interv 2018;92:1309-1314.

Table

Table 1. Clinical and Echocardiographic Data

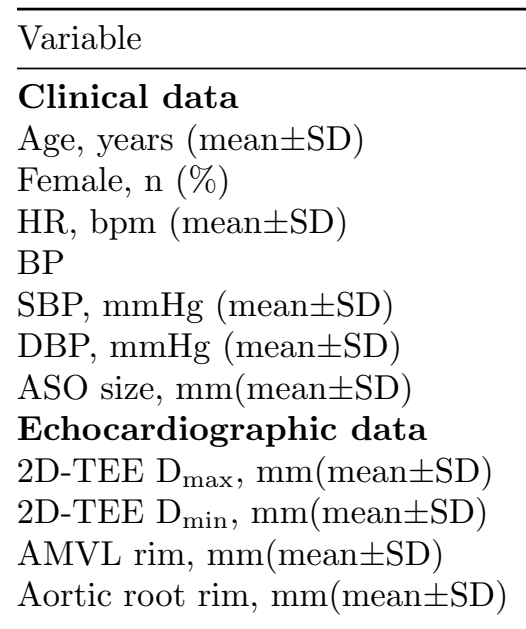


Variable

SVC rim, $\mathrm{mm}($ mean $\pm \mathrm{SD})$

IVC rim, $\mathrm{mm}($ mean $\pm \mathrm{SD})$

Oval ASD, n (\%)

Round ASD, n (\%)

double-hole, secundum ASD, n (\%)

isolated secundum ASD, n (\%)

$\mathrm{LAD}, \operatorname{mm}($ mean $\pm \mathrm{SD})$

RAD-ml, mm(mean \pm SD) RAD-si, mm $($ mean \pm SD)

RVD-ap, $m m(m e a n \pm S D)$ RVD-bml, mm(mean \pm SD) RVD-mml, mm(mean \pm SD) RVD-si, mm(mean \pm SD) TAPSE, mm(mea

LVEDd, $\mathrm{mm}($ mean $\pm \mathrm{SD})$ TVR-Vmax, $\mathrm{cm} / \mathrm{s}($ mean $\pm \mathrm{SD}) \mathrm{TVR}-\mathrm{PG}, \mathrm{mmHg}($ mean $\pm \mathrm{SD}) \mathrm{RAP}, \mathrm{mmHg}(\operatorname{mean} \pm \mathrm{SD})$

$\mathrm{PASP}, \mathrm{mmHg}($ mean $\pm \mathrm{SD})$

$\operatorname{LVEF}(\%)$

Abbreviations: HR, heart rate; BP, blood pressure; SBP, systolic blood pressure; DBP, diastole blood pressure; ASO, Amplatzer septal occluder; 2D-TEE, two-dimensional transesophageal echocardiography ; $\mathrm{D}_{\max }$, the maximal diameter; $\mathrm{D}_{\min }$, the minimum diameter; AMVL, anterior mitral valve leaflet; SVC, superior vena cava; IVC, inferior vena cava; ASD, atrial septal defect; LAD, left atrium dimension; RAD-ml, mediallateral right atrium dimension; RAD-si, superior-inferior right atrium dimension; RVD-ap, antero-posterior right ventricular dimension; RVD-bml, basal medial-lateral right ventricular dimension; RVD-mml, middle medial-lateral right ventricular dimension; RVD-si, superior-inferior right ventricular dimension; TAPSE, tricuspid annulu plane systolic excusion; LVEDd, left ventricular end diastolic dimension; TVR- Vmax, the maximal velocity of tricuspid valve regurgitation ;PG, pressure gradient; RAP, right atrial pressure; PASP, pulmonary arterial systolic pressure; LVEF, left ventricular ejection fraction.

\section{Hosted file}

image1.wmf available at https://authorea.com/users/444222/articles/544019-three-dimensionalprinting-of-atrial-septal-defect-from-echocardiographic-images-feasibility-fidelity-andpotential-applications

Table 2 Comparison of ASD Parameters between3D Printed Models and 2D-TEE Images ()

\begin{tabular}{llll}
\hline Methods & $\begin{array}{l}\text { The maximum } \\
\text { diameter }(\mathrm{mm})\end{array}$ & $\begin{array}{l}\text { The minimum diameter } \\
(\mathrm{mm})\end{array}$ & Circular index \\
3D printed models & $20.22 \pm 4.53$ & $14.65 \pm 3.66$ & $1.40 \pm 0.23$ \\
2D-TEE images & $20.14 \pm 4.42$ & $14.58 \pm 3.71$ & $1.40 \pm 0.20$ \\
$t$ value & 0.76 & 0.72 & -0.10 \\
$P$ value & 0.45 & 0.47 & 0.92 \\
\hline
\end{tabular}

Abbreviations: ASD, atrial septal defect;3D, three dimensional; 2D-TEE, two-dimensional transesophageal echocardiography. 


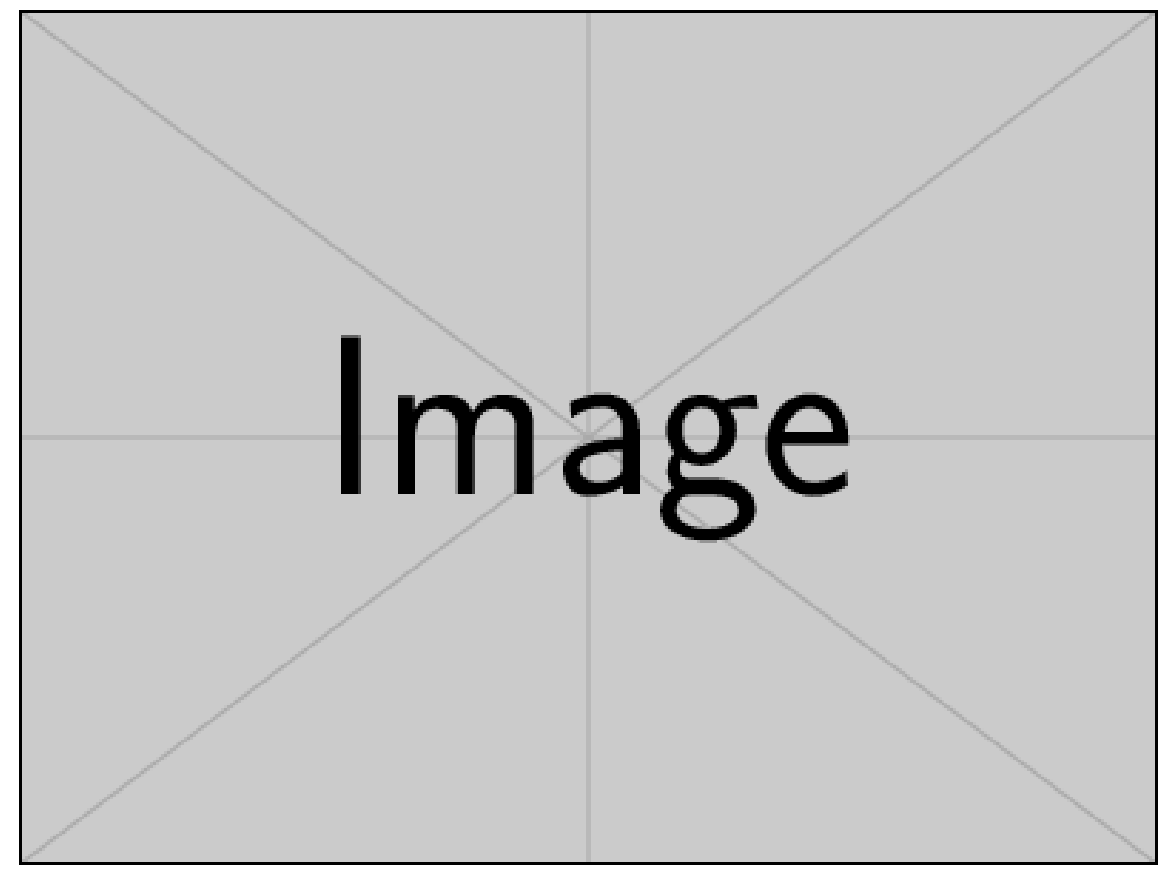

Table 3 Comparison of ASD parameters between 3D printed models and 3D-TEE Images ()

\begin{tabular}{lllll}
\hline Methods & $\begin{array}{l}\text { The maximum } \\
\text { diameter }(\mathrm{mm})\end{array}$ & $\begin{array}{l}\text { The minimum } \\
\text { diameter }(\mathrm{mm})\end{array}$ & Circular index & $\begin{array}{l}\text { Circumference } \\
(\mathrm{mm})\end{array}$ \\
$\begin{array}{l}\text { 3D printed } \\
\text { models }\end{array}$ & $\begin{array}{l}14.65 \pm 3.66 \\
\text { 3D-TEE images }\end{array}$ & $20.22 \pm 4.53$ & $1.40 \pm 0.23$ & $56.13 \pm 12.00$ \\
$t$ value & -1.22 & $14.71 \pm 3.85$ & $1.41 \pm 0.22$ & $56.40 \pm 12.10$ \\
$P$ value & 0.23 & -0.52 & -0.50 & -1.23 \\
\hline
\end{tabular}

Abbreviations: ASD, atrial septal defect;3D, three dimensional;3D-TEE, three-dimensional transesophageal echocardiography.

Table 4 The 3D printed ASD size parameters and the intraoperative occluder size parameters

\begin{tabular}{llllll}
\hline Patient no. & $\begin{array}{l}3 \mathrm{DPM} \mathrm{D}_{\max } \\
(\mathrm{mm})\end{array}$ & $\begin{array}{l}3 \mathrm{DPM} \mathrm{D}_{\min } \\
(\mathrm{mm})\end{array}$ & $3 \mathrm{DPM} \mathrm{C}(\mathrm{mm})$ & ASO WD $(\mathrm{mm})$ & ASO C $(\mathrm{mm})$ \\
\hline 1 & 26.31 & 16.72 & 73.00 & 34.00 & 106.81 \\
2 & 12.80 & 10.50 & 41.00 & 20.00 & 62.83 \\
3 & 26.91 & 17.30 & 73.00 & 34.00 & 106.81 \\
4 & 19.90 & 19.12 & 58.10 & 28.00 & 87.96 \\
5 & 22.64 & 12.87 & 61.00 & 30.00 & 94.25 \\
6 & 9.98 & 7.06 & 24.88 & 18.00 & 56.55 \\
7 & 15.13 & 9.28 & 37.57 & 26.00 & 81.68 \\
8 & 15.09 & 11.50 & 46.00 & 22.00 & 69.11 \\
9 & 21.20 & 13.88 & 58.06 & 30.00 & 94.25 \\
10 & 16.80 & 13.50 & 47.39 & 22.00 & 69.11 \\
11 & 27.39 & 23.21 & 75.11 & 38.00 & 119.38
\end{tabular}




\begin{tabular}{llllll}
\hline Patient no. & $\begin{array}{l}3 \mathrm{DPM} \mathrm{D}_{\max } \\
(\mathrm{mm})\end{array}$ & $\begin{array}{l}3 \mathrm{DPM} \mathrm{D}_{\min } \\
(\mathrm{mm})\end{array}$ & $3 \mathrm{DPM} \mathrm{C}(\mathrm{mm})$ & ASO WD $(\mathrm{mm})$ & ASO C $(\mathrm{mm})$ \\
\hline 12 & 20.30 & 16.58 & 56.71 & 30.00 & 94.25 \\
13 & 19.18 & 14.05 & 49.65 & 26.00 & 81.68 \\
14 & 23.90 & 18.06 & 68.21 & 32.00 & 100.53 \\
15 & 23.36 & 14.90 & 57.48 & 30.00 & 94.25 \\
16 & 20.69 & 16.37 & 57.51 & 30.00 & 94.25 \\
17 & 23.45 & 15.93 & 63.80 & 30.00 & 94.25 \\
18 & 26.08 & 15.40 & 67.12 & 30.00 & 94.25 \\
19 & 24.66 & 18.50 & 69.02 & 30.00 & 94.25 \\
20 & 12.75 & 11.44 & 40.72 & 20.00 & 62.83 \\
21 & 17.27 & 10.51 & 46.22 & 22.00 & 69.11 \\
22 & 22.30 & 11.62 & 57.62 & 26.00 & 81.68 \\
23 & 17.35 & 10.93 & 46.15 & 20.00 & 62.83 \\
24 & 15.65 & 12.04 & 46.4 & 24.00 & 75.40 \\
25 & 22.62 & 20.67 & 71.19 & 32.00 & 100.53 \\
26 & 13.83 & 10.88 & 39.67 & 20.00 & 62.83 \\
27 & 18.90 & 10.57 & 51.12 & 20.00 & 62.83 \\
28 & 15.08 & 9.58 & 40.47 & 22.00 & 69.11 \\
29 & 21.52 & 14.45 & 55.41 & 30.00 & 94.25 \\
30 & 20.89 & 12.49 & 51.87 & 24.00 & 75.40 \\
31 & 22.23 & 13.06 & 56.95 & 28.00 & 87.96 \\
32 & 27.83 & 18.34 & 75.15 & 34.00 & 106.81 \\
33 & 18.82 & 16.68 & 58.34 & 28.00 & 87.96 \\
34 & 16.78 & 14.09 & 49.89 & 22.00 & 69.11 \\
35 & 20.58 & 14.10 & 54.90 & 26.00 & 81.68 \\
36 & 15.31 & 12.95 & 49.18 & 22.00 & 69.11 \\
37 & 21.28 & 18.47 & 60.47 & 34.00 & 106.81 \\
38 & 28.35 & 19.26 & 75.00 & 36.00 & 113.09 \\
39 & 22.09 & 19.21 & 65.83 & 32.00 & 100.53 \\
40 & 21.43 & 20.07 & 68.14 & 34.00 & 106.81 \\
\hline & & & & & \\
& & & & & \\
\end{tabular}

Abbreviations: 3DPM, three-dimensional printed model; $\mathrm{D}_{\max }$, the maximal diameter; $\mathrm{D}_{\min }$, the minimum diameter; C,circumference; ASO, Amplatzer septal occlude;WD, waist diameter

Table 5 Simulation operation results

\begin{tabular}{|c|c|c|c|c|c|c|c|c|}
\hline Patient no. & ASD type & $\begin{array}{l}\text { Shape of } \\
\text { ASD }\end{array}$ & $\mathrm{D}_{\max }(\mathrm{mm})$ & $\mathrm{D}_{\min }(\mathrm{mm})$ & CI & $\begin{array}{l}\text { Occluder } \\
\text { replacement }\end{array}$ & $\begin{array}{l}\text { Simulate } \\
\text { operation } \\
\text { occluder } \\
\text { size }(\mathrm{mm})\end{array}$ & $\begin{array}{l}\text { Intraop } \\
\text { occlude } \\
\text { size }(\mathrm{mr}\end{array}$ \\
\hline 1 & $\begin{array}{l}\text { One- } \\
\text { hole }\end{array}$ & Round & 16.80 & 13.50 & 1.24 & No & 22 & 22 \\
\hline 2 & $\begin{array}{l}\text { One- } \\
\text { hole }\end{array}$ & Round & 22.62 & 20.67 & 1.09 & Yes & 28 & 32 \\
\hline 3 & $\begin{array}{l}\text { One- } \\
\text { hole }\end{array}$ & Oval & 26.91 & 17.30 & 1.56 & No & 34 & 34 \\
\hline 4 & $\begin{array}{l}\text { One- } \\
\text { hole }\end{array}$ & Oval & 17.35 & 10.93 & 1.59 & No & 20 & 20 \\
\hline
\end{tabular}




\begin{tabular}{lllllllll}
\hline & & & & & & & $\begin{array}{l}\text { Simulate } \\
\text { operation } \\
\text { occluder } \\
\text { size }(\mathrm{mm})\end{array}$ & $\begin{array}{l}\text { Intraop } \\
\text { occlude } \\
\text { size }(\mathrm{mn}\end{array}$ \\
Patient no. & ASD type & $\begin{array}{l}\text { Shape of } \\
\text { ASD }\end{array}$ & $\mathrm{D}_{\max }(\mathrm{mm})$ & $\mathrm{D}_{\min }(\mathrm{mm})$ & CI & $\begin{array}{l}\text { Occluder } \\
\text { replacement }\end{array}$ \\
\hline 5 & $\begin{array}{l}\text { duoble- } \\
\text { hole }\end{array}$ & Round & 21.28 & 18.47 & 1.15 & Yes & 30 & 34 \\
\hline
\end{tabular}

Abbreviations: ASD, atrial septal defect; $\mathrm{D}_{\max }$, the maximal diameter; $\mathrm{D}_{\min }$, the minimum diameter; $\mathrm{CI}$; circular index

\section{Figure Legends}

Fig 1 . The workflow of three-dimensional (3D) printing of a three-dimensional transesophageal echocardiography (3D TEE) derived atrial septal defect (ASD)model.

A. After Acquiring the high-quality 3D-TEE image, the data set was exported from the ultrasound system to the EchoPAC workstation anonymously and converted to standard DICOM format.

B. The DICOM format of optimized 3D-TEE ASD image at the isovolumetric diastole stored in the EchoPAC workstation was imported into the Mimics software to label the region of interest and create a 3D digital ASD model.

C. The 3D digital ASD model was converted to a 3D hollow mold by software 3-matic.

D. The soft 3D printed ASD model generated by rubber like material.

Fig 2. Three types of 3D printed ASD models.

A. The round one-hole ASD model, of which the maximal diameter, the minimum diameter, circular index and circumference measured from the 3D printed ASD model was 22.62mm, 20.67mm, 1.09,71.19mm, respectively.

B. The oval one-hole ASD model, of which the maximal diameter, minimum diameter, circular index and circumference measured from the 3D printed ASD model was $26.91 \mathrm{~mm}, 17.30 \mathrm{~mm}, 1.56$, and $73.00 \mathrm{~mm}$, respectively.

C. The double-hole ASD model, the size of the two holes are $16.09 \times 10.00 \mathrm{~mm}$ and $6.68 \times 5.17 \mathrm{~mm}$. The distance between the two defects is $6 \mathrm{~mm}$, and the whole size of the ASD is $21.28 \times 18.47 \mathrm{~mm}$.

Fig 3. Simulation exercises

On the basis of comprehensive analysis of the size parameters of ASD models, simulation exercises are performed in the models, the most suitable device finally selected according to the simulation operation is confirmed to be the same as the one selected in actual surgical procedure and the preoperative exercises achieved good results.

A. In the round one-hole ASD model, a 32mm Amplatzer septal occluder (ASO) was selected after two attempts.

B. In the oval one-hole ASD case, a 34mm ASO was applied to occlude the defect successfully.

C. In the biforate ASD model, a $34 \mathrm{~mm}$ ASO was finally selected to cover all the defects successfully with the bigger defect as the optimal target defect after several experiments in vitro.

Fig 4. Bland-Altman plots showing the agreement of atrial septal defect (ASD)size parameters as the maximal diameter $\left(\mathrm{D}_{\max }\right)(\mathrm{A})$, the minimum diameter $\left(\mathrm{D}_{\min }\right)(\mathrm{B})$, circular index $(\mathrm{C})$, and circumference $(\mathrm{D})$ between the three-dimensional transesophageal echocardiography(3D-TEE) images and three-dimensional 
printed models(3DPM). The solid lines represent average differences of the two methods, and dashed lines represent the limitation of agreement.

Fig 5 . Bland-Altman plots showing the agreement of atrial septal defect (ASD)size parameters as the maximal diameter $\left(\mathrm{D}_{\max }\right)(\mathrm{A})$, the minimum diameter $\left(\mathrm{D}_{\min }\right)(\mathrm{B})$, circular index $(\mathrm{C})$ between the two-dimensional transesophageal echocardiography(2D-TEE) images and three-dimensional printed models(3DPM). The solid lines represent average differences of the two methods, and dashed lines represent the limitation of agreement.
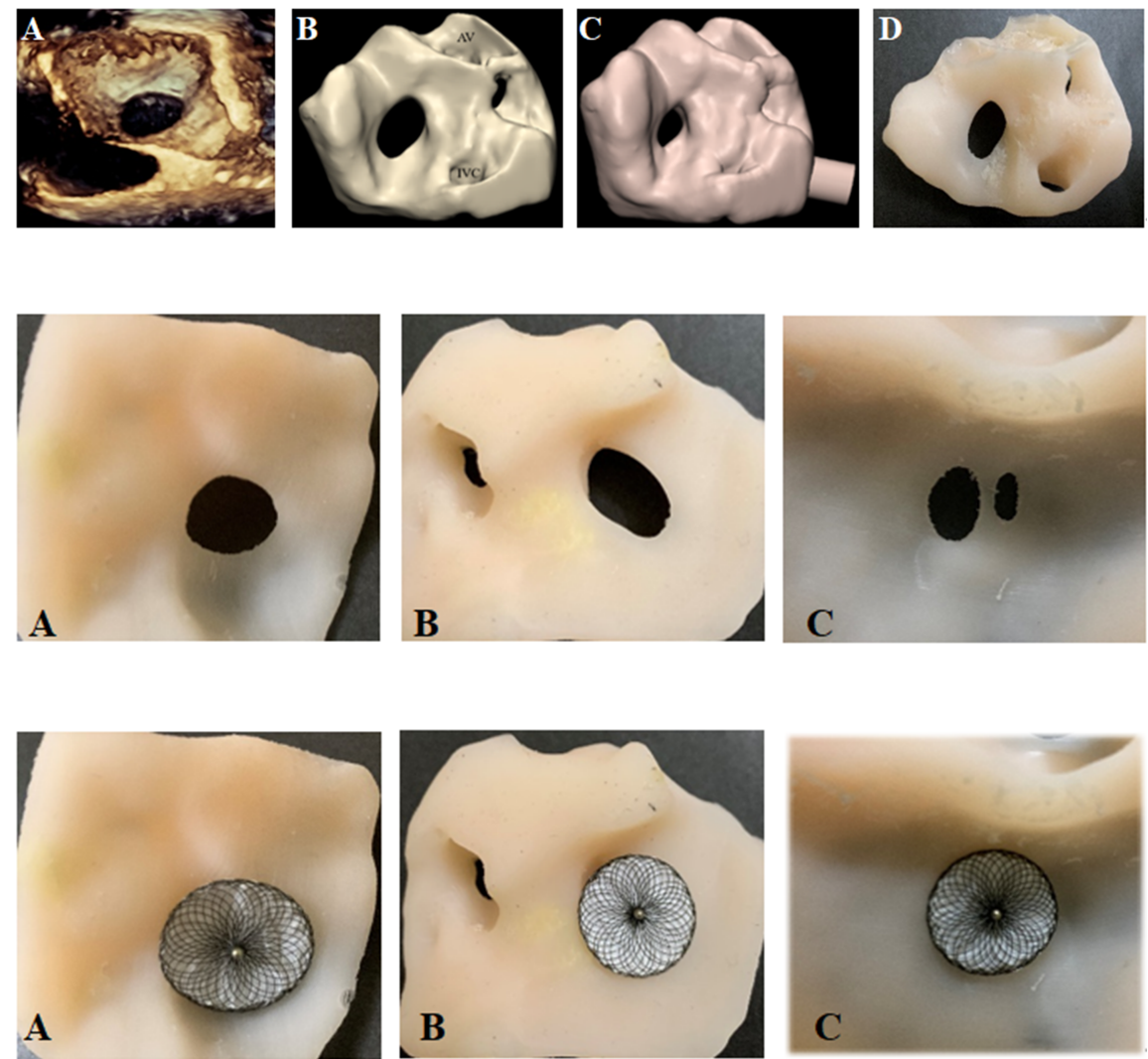

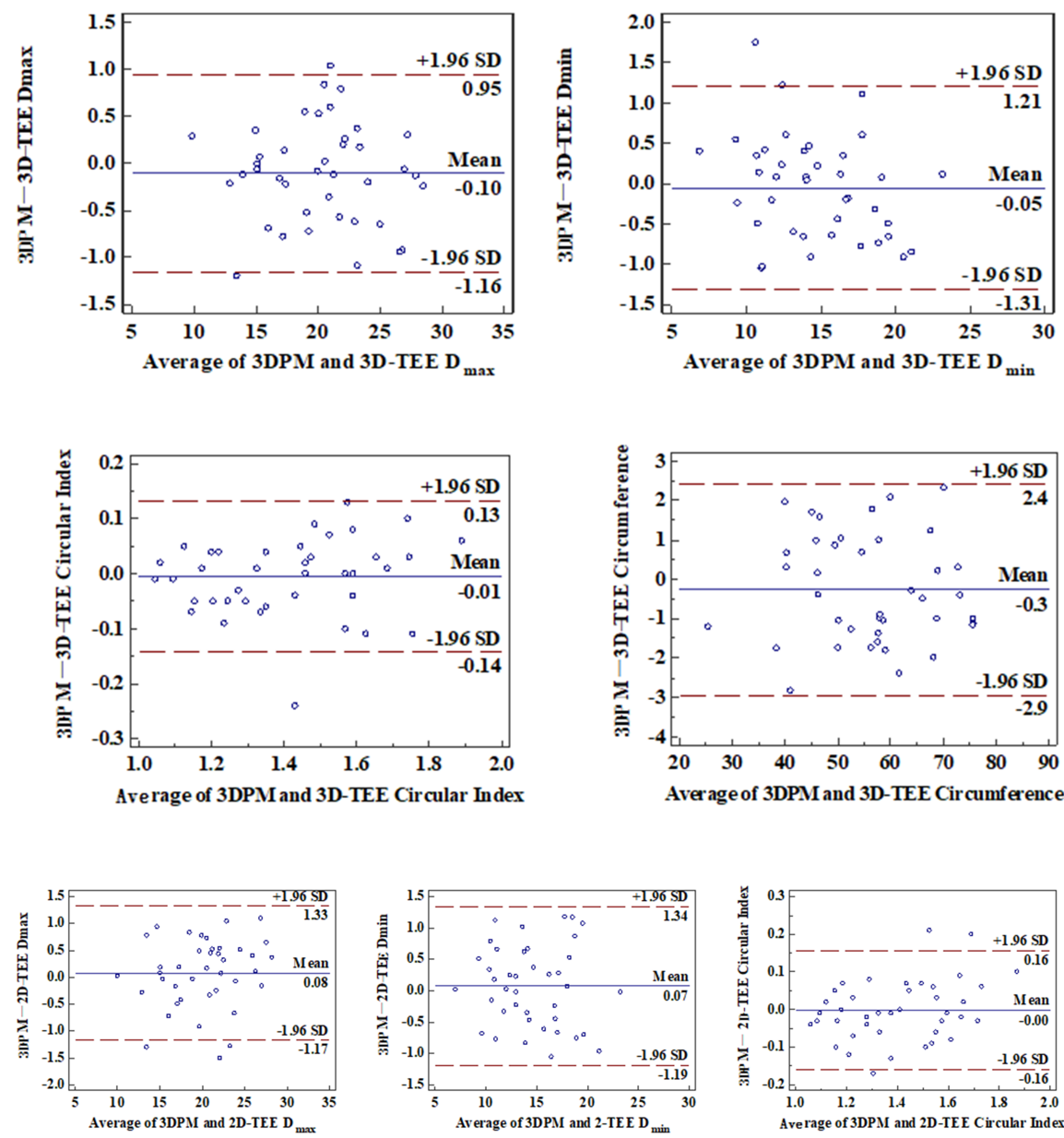

\section{Hosted file}

table.docx available at https://authorea.com/users/444222/articles/544019-three-dimensionalprinting-of-atrial-septal-defect-from-echocardiographic-images-feasibility-fidelity-andpotential-applications 